\title{
Erosão dentária em paciente infantil: um relato de caso
}

\author{
Dental erosion in pediatric dentistry: a case report \\ Erosión dental en odontología pediátrica: reporte de un caso
}

\begin{abstract}
Ana Paula de Almeida Nunes ${ }^{1 *}$, Natália de Oliveira Moreira Bechtlufft ${ }^{1}$, Mariana Santos Delgado ${ }^{1}$, Camila Faria Carrada'.
\end{abstract}

\section{RESUMO}

Objetivo: Relatar um caso clínico de paciente infantil com presença de erosão dentária associada ao consumo excessivo de alimentos ácidos. Detalhamento do Caso: Paciente, F.E.R. 11 anos, sexo masculino, compareceu à clínica-escola de odontopediatria, em uma instituição de ensino em Odontologia. Durante exame clínico observouse lesões generalizadas sugestivas de erosão dentária, com as seguintes características: brilho excessivo, lisura superficial, esmalte hígido ao longo da margem gengival, além de cuppings e halo translúcido ao redor da superfície oclusal/incisal de molares, pré-molares, caninos e lingual/palatina de incisivos permanentes. Afim de descobrir os fatores etiológicos da doença, foi empregado um diário alimentar para a criança, recordatório de 24 horas. 0 questionário aplicado demonstrou consumo excessivo de alimentos ácidos. Considerações Finais: Diante do exposto, o tratamento proposto foi orientação aos responsáveis e à criança sobre a erosão dentária e sua relação com a dieta de alimentos ácidos e adoção de medidas para o impedimento da progressão da doença, como aplicação de verniz fluoretado. O diagnóstico precoce das lesões minimiza os danos estruturais no elemento dental e favorece o tratamento desta condição bucal.

Palavras-chave: Erosão dentária, Odontopediatria, Desgaste dos dentes.

\section{ABSTRACT}

Objective: To report a clinical case of a child patient with the presence of dental erosion associated with excessive consumption of acidic foods. Case Details: Patient, F.E.R. 11 years old, male, attended the pediatric dentistry school clinic, in an educational institution in Dentistry. During clinical examination, generalized lesions suggestive of dental erosion were observed, with the following characteristics: excessive shine, superficial smoothness, healthy enamel along the gingival margin, in addition to cuppings and translucent halo around the occlusal / incisal surface of molars and canines and lingual / palatal permanent incisors. In order to discover the etiological factors of the disease, a food diary for the child was used, a 24-hour recall. The applied questionnaire showed excessive consumption of acidic foods. Final Cosiderations: In view of the above, the proposed treatment was guidance to parents and children about dental erosion and its relationship with the diet of acidic foods and adoption of measures to prevent the progression of the disease, such as the application of fluoride varnish. of the lesions minimizes the structural damage in the dental element and favors the treatment of this oral condition.

Key words: Tooth erosion, Pediatric dentistry, Tooth wear.

\section{RESUMEN}

Objetivo: Informar un caso clínico de un paciente infantil con la presencia de erosión dental asociada con el consumo excesivo de alimentos ácidos. Detalles del Caso: Paciente, F.E.R. 11 años, varón, asistió a la clínica de la escuela de odontopediatría, en una institución educativa en Odontología. Durante el examen clínico, se observaron lesiones generalizadas sugestivas de erosión dental, con las siguientes características: brillo excesivo, suavidad superficial, esmalte saludable a lo largo del margen gingival, además de ventosas y halo translúcido alrededor de la superficie oclusal / incisal de los molares y caninos e incisivos linguales / palatales permanentes. Para descubrir los factores etiológicos de la enfermedad, se utilizó un diario de alimentos para el niño, un recuerdo de 24 horas. El cuestionario aplicado mostró un consumo excesivo de alimentos ácidos. Consideraciones Finales: En vista de lo anterior, el tratamiento propuesto fue una guía para padres e hijos sobre la erosión dental y su relación con la dieta de alimentos ácidos y la adopción de medidas para prevenir la progresión de la enfermedad, como la aplicación de barniz de flúor. de las lesiones minimiza el daño estructural en el elemento dental y favorece el tratamiento de esta afección oral.

Palabras clave: Erosión de los dientes, Odontología pediátrica, Desgaste de los dientes.

${ }^{1}$ Faculdade de Ciências Médicas e da Saúde de Juiz de Fora, Juiz de Fora - MG

*E-mail: anapaulalmeidanunes@yahoo.com.br

SUBMETIDO EM: 12/2020 


\section{INTRODUÇÃO}

A erosão dentária tem por definição perda patológica física da superfície dentária, causada por ataque químico e/ou quelante, podendo ser crônica, assintomática, localizada onde não há envolvimento de microorganismos (SALAS MM, et al., 2015). A causa provável dessa desmineralização é o ácido de origem não bacteriano, onde é removido a matriz orgânica do elemento dentário (CARVALHO TS, et al., 2015; CRISSI PSO, et al., 2014).

A comunidade científica tem dado grande importância e maior reconhecimento para estas lesões pelo fato do aumento da incidência em crianças e adolescentes (SANTANA MNS, et al., 2008). Tem por características clínica a erosão dentária, uma superfície lisa e brilhante, fosca e transparente, o esmalte com borda intacta, próximo a margem gengival. Quando houver depressões e superfícies côncavas, sobrecontorno nas restaurações de amálgama e mais raramente exposição pulpar, mostra ser a forma mais severa (BARTIETT D, et al., 2008).

Essa erosão pode ser proveniente do ácido dos alimentos que são ingeridos na dieta, fontes intrínsecas e não da flora intrabucal. Segundo Imfeld (1996) as classificações podem ser divididas baseada na sua etiologia: Erosão extrínseca: sendo por ácidos exógenos; Erosão intrínseca: sendo por ácidos endógenos. Envolve dentina em mais de um terço da superfície. Lesões generalizadas. III-a: superfícies vestibulares; IIIb: superfícies linguais e palatinas; III-c: superfícies incisais e oclusais; III-d: múltiplas superfícies com comprometimento severo (ALVES MSC, et al., 2012).

A etiologia da erosão está ligada a fatores extrínsecos, sendo os ácidos da dieta o principal deles, presentes nos alimentos e bebidas em especial frutas e seus sucos e o refrigerante, neles podem estar presentes variados ácidos danosos ao dente. Tem também os ácidos do ambiente de trabalho assim como piscinas com pH deficientemente monitorado o uso de por via oral de medicamentos erosivos. Então sendo esses os de fontes extrínsecas (AL-DLAIGAN YH, et al., 2017)

Outro fator causador da erosão dentária são os ácidos endógenos ácidos provenientes do refluxo voluntário ou involuntário. Em pacientes com desordem psicossomáticas, como a bulimia e anorexia geralmente em pacientes jovens do sexo feminino de idades entre vinte e trinta anos. $\mathrm{E}$ as desordens somáticas ligado ao alcoolismo, problemas gastrointestinais, vômitos, regurgitação, recorrentes quadros de refluxos e o período gestacional. E por fim a erosões cujo o diagnóstico não é possível por exame clínico ou de anamnese, denominada idiopática (FONTES CL, et al., 2016; LUSSI A e JAEGGI T, 2008).

Como forma de tratamento, o primeiro passo é a eliminação do efeito causal, no intuito de prevenir a evolução e o aparecimento de novas lesões. O cirurgião-dentista tem o papel de reconhecer, diagnosticar, orientar, tratar e acompanhar regularmente a lesão, a fim de promover uma estabilização desse desgaste que pode acontecer de forma rápida e progressiva. Dependendo do grau de desgaste, os tipos de tratamento serão indicados desde aplicações de verniz fluoretado a procedimentos restauradores convencionais, diretos ou indiretos acompanhados de mudança de hábitos comportamentais do indivíduo (BRANCO CA, et al., 2008).

Sendo assim, o presente trabalho teve como objetivo relatar o caso clínico de paciente infantil com erosão dentária.

\section{DETALHAMENTO DO CASO}

Paciente, F.E.R. 11 anos, sexo masculino, compareceu à clínica de Odontopediatria de uma instituição de ensino em Odontologia, para primeiro atendimento. Inicialmente foi realizado o preenchimento completo da anamnese e do termo de consentimento livre e esclarecido, com acompanhamento do responsável, que relatou a nível de complemento dos dados clínicos para histórica médica da criança, que a mesma estava em acompanhamento psicológico e que a adoção a família era recente, e dessa forma dados anteriores sobre a história médica não saberiam relatar. Durante exame clínico observou-se lesões sugestivas de erosão dentária, paciente apresentava leve sensibilidade dolorosa e apresentava também desgaste incisal/oclusal bilateralmente, sem guias laterais. 
Aparência de brilho excessivo, lisura superficial, esmalte hígido ao longo da margem gengival, além de cuppings e halo translúcido ao redor da superfície oclusal/incisal de molares, pré-molares, caninos e lingual/palatina de incisivos permanentes. Na Figura 1, observamos os cuppings em face oclusal de prémolares e molares permanentes.

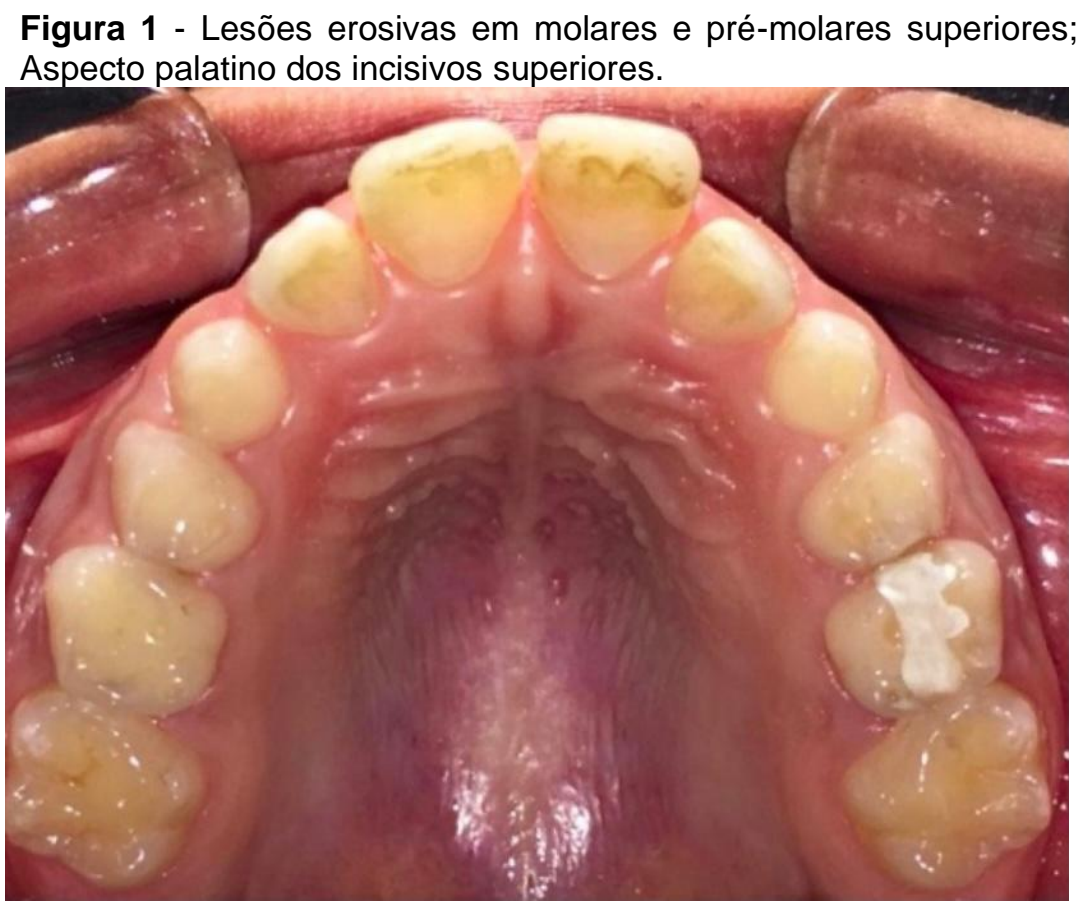

Fonte: Nunes APA, et al., 2020.

Aspecto da arcada inferior, diagnosticada com escore 2, no índice Basic Erosive Wear Examination (BEWE), entretanto apenas nos pré-molares, molares decíduos e molares permanentes (Figura 2A). Lisura superficial derivada do desgaste erosivo (Figura 2B).

Figura 2 - Lesões erosivas em molares e pré-molares inferiores (A); Aspecto cuppings oclusais (B).
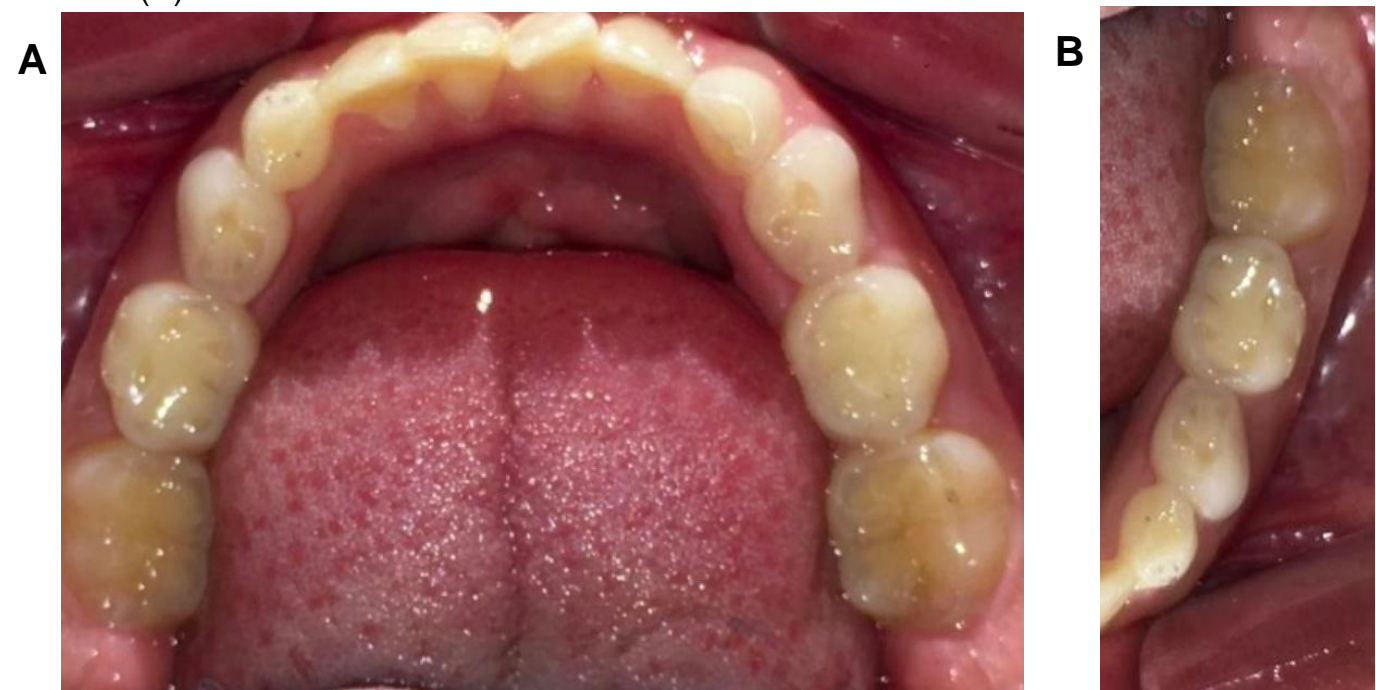

Fonte: Nunes APA, et al., 2020.

Na Figura 3A e B, observamos o desgaste incisal/oclusal de canino decíduo, pré-molares e molares permanentes, bem como molar decíduo. Mostrando na imagem incisivos centrais superiores vestibularizados, com overjet. 
Figuras 3 - Desgaste oclusal em molares e perda de guia canina, bilateralmente.
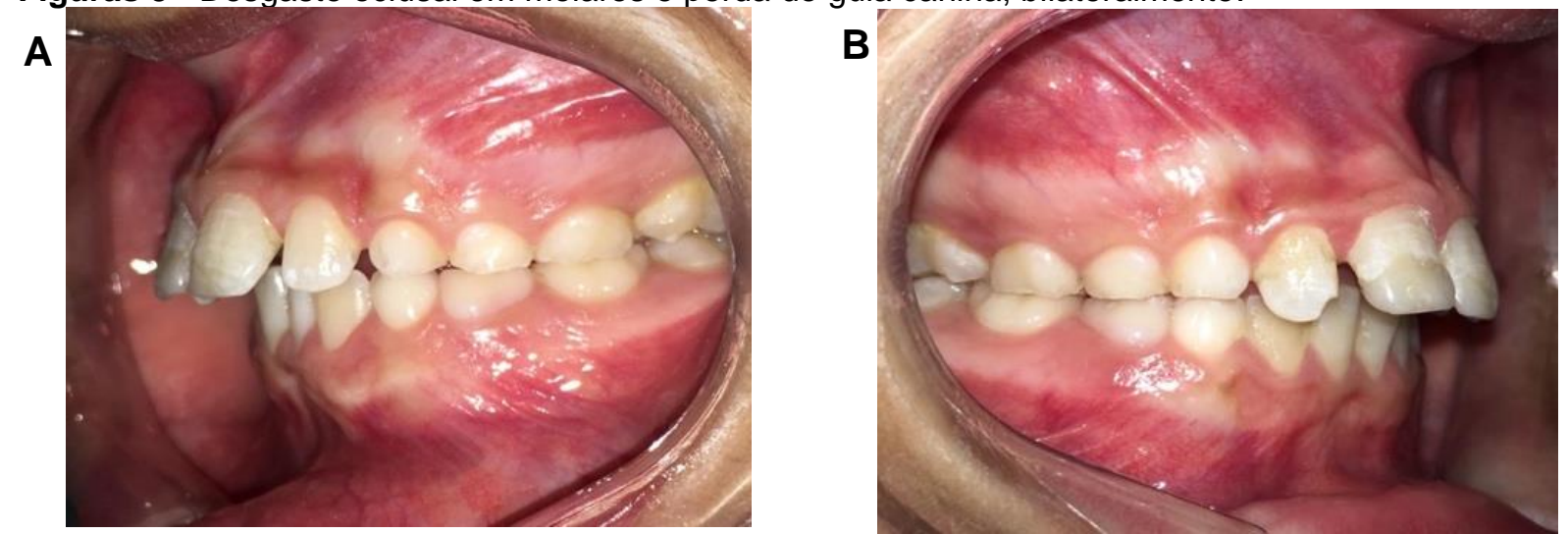

Fonte: Nunes APA, et al., 2020.

Diante do diagnóstico obtido, iniciou-se um processo de investigação afim de descobrir os fatores etiológicos da doença, foi empregado um diário alimentar para a criança, recordatório de 24 horas, durante as consultas subsequentes para descobrir a origem da erosão (extrínseca ou intrínseca). De acordo com as respostas obtidas nos questionamentos, foi identificado o consumo excessivo de refrigerante à base de cola, e de frutas cítricas, principalmente o abacaxi, confirmado pelo responsável da criança, e além da frequente mastigação de balas ácidas.

Para correta escolha terapêutica, os elementos dentários foram submetidos a uma profilaxia com escova de robinson e pasta profilática, para serem analisados atráves do Escore Bewe (Basic Erosive Wear Examination) para identificação dos sinais clínicos da erosão em relação ao grau de severidade, onde o paciente foi classificado no escore 2, com perda do tecido em menos de $50 \%$ da área de superfície em dentina, apontando-o como sendo de médio risco, visto que sua contabilização parcial de sextantes foi igual a 12, na classificação de critérios de desgaste.

De acordo com o índice BEWE, e seguindo os padrões estabelecidos com o resultado encontrado do paciente, orientou-se os responsáveis e ao paciente, a prática de uma boa higiene oral, aguardar o período de vinte minutos para realizar a escovação dental, além de manter uma dieta saudável, reduzindo significativamente a taxa de ingestão de alimentos e bebidas ácidas. Foi realizada, então, sob isolamento relativo, a aplicação semanal de verniz de fluoreto de sódio 5\% (Fluorniz,SS White, Brasil), nas áreas dos dentes submetidos à erosão para que as superfícies dentárias apresentassem maior resistência, e a orientação de continuidade no acompanhamento odontológico para controle da progressão e sintomas.

\section{DISCUSSÃO}

No presente estudo foi também aplicado um questionário recordatório, nas consultas subsequentes, capaz de identificar as principais causas da erosão, e fatores que poderiam influenciar no desgaste dentário, como hábitos de higiene oral, força de escovação e saúde geral.

A prevalência do desgaste dentário erosivo tem aumentado, principalmente em crianças e adolescentes (COMAR LP, et al., 2013). Uma revisão sistemática indicou que a prevalência de desgaste envolvendo dentina variou em até $82 \%$ e $54 \%$ para dentes decíduos e dentes permanentes, respectivamente, em crianças de até 7 anos de idade (USHIDA CE e CURY JÁ, 1999). Corroborando com esses resultados, uma amostra aleatória de 1035 crianças de 14 anos de idade mostrou que 30\% apresentavam dentina exposta na incisal e 8\% exposição em superfícies oclusais e/ou lingual (COMAR LP, et al., 2013).

Outro estudo realizado com 101 crianças de 4 a 16 anos de idade encontrou a exposição da dentina em $30 \%$ dos molares decíduos (BRUSIUS CD, 2013). Uma das principais razões pelas quais houve esse aumento, são as mudanças dos hábitos dietéticos da população, que tem consumido mais produtos industrializados, como os refrigerantes e bebidas isotônicas, o que pôde ser constatado pelos dados da pesquisa de orçamento familiar que apontou que, entre os anos de 1975 a 2003, a aquisição per capita de 
refrigerantes aumentou de 1,29 para 7,65 , o que equivale a $490 \%$ de crescimento (CORRÊA FNP, et al., 2011).

As lesões de erosão dentária podem variar de acordo com sua severidade (KREULEN CM, et al., 2010). Algumas podem se restringir à superfície do esmalte, enquanto outras podem apresentar perda de superfície do esmalte e até a exposição de dentina (KREULEN CM, et al., 2010; LINNETT V e SEOW WK, 2001). A maioria dos estudos analisados concluem que as lesões de erosão em crianças e adolescentes acometem apenas o esmalte, e poucos avaliaram o desgaste a nível de dentina. Isso acontece devido ao curto tempo de intervenção dos fatores etiológicos sob a estrutura dentária em crianças e adolescentes (MESSIAS DCF, et al., 2011).

O Escore BEWE, com o objetivo de diagnóstico e conduta do tratamento de lesões erosivas, é um sistema para registrar a superfície mais severa afetada em um sextante por meio da contabilização parcial (LINNETT V e SEOW WK, 2001). Nesse intuito, avalia-se as superfícies vestibulares, oclusal e lingual/palatal em todos os dentes de cada sextante, porém registra-se somente o resultado da superfície do dente que tiver maior valor. Em questão ao caso, paciente apresentava escore 2, com presença de defeito distinto com perda de tecido duro menor que 50\% da superfície (KREULEN CM, et al., 2010; LINNETT V e SEOW WK, 2001).

$\mathrm{O}$ aumento do consumo de refrigerantes e bebidas esportivas entre crianças e adolescentes pode estar relacionado às mudanças nos estilos de vida, bem como melhores condições financeiras e maior disponibilidade de bebidas industrializadas ácidas. Porém, evidencia-se que a saliva como sustância de tampão pode intervir na presença ou não de erosão dentária, mesmo com a frequente ingestão de alimentos ou bebidas ácidas (RANDAZZO AR, et al., 2020).

Por outro lado, a literatura diverge quando o assunto é a possibilidade da relação entre erosão dentária e nível socioeconômico das crianças. Apesar de o nível socioeconômico não apresentar efeito total sobre a erosão, existem estudos que apresentam dados de que crianças mais favorecidas economicamente possuem significativamente menos erosão do que as de outros grupos. Além disso, foi observado que crianças com menor poder socioeconômico tiveram maior prevalência da lesão. Porém, uma hipótese que pode explicar as divergências entre os estudos realizados se baseia no acesso que as crianças e adolescentes têm a bebidas e comidas ácidas (RANDAZZO AR, et al., 2020; MAGALHÃES AC, 2008).

Estudos mostram que quanto maior a idade do paciente, maior a prevalência da erosão dentária e maior a possibilidade de a lesão ser severa. Assim, o tempo de exposição pode ser considerado um dos fatores que mais influenciam na ocorrência da erosão, visto que crianças mais velhas tem maior exposição ao estímulo erosivo. Além disso, tanto nos estudos de prevalência quanto nos de incidência, os resultados apresentaram que a severidade das lesões na população infantil foi limitada ao esmalte, mas com o tempo pode atingir a dentina. Assim, crianças mais velhas tendem a apresentar lesões mais avançadas e severas (MAGALHÃES AC, 2008).

Dessa maneira, não existem muitos estudos longitudinais que avaliaram a erosão. Pesquisas feitas por Dugmore CR e Rock WS, (2004) e El Aidi H, et al. (2010) acompanharam, durante 2 a 3 anos, crianças e adolescentes, observaram que, dos 12 aos 14 anos de idade, houve aumento da incidência de erosão dentária, enquanto El Aidi $\mathrm{H}$, et al., (2010) documentou que ocorreu um aumento da erosão até o segundo ano de avaliação, mas após esse período, houve estabilização.

Existem diversos estudos que mostram que crianças do sexo masculino apresentam maior incidência de erosão dentária do que do sexo feminino. Segundo Bardolia P, et al., (2010), maior porcentagem de meninos teve a dentina exposta em superfícies lisas oclusais em relação às meninas, e a força de mordida poderia ser uma explicação para a diferença da prevalência de erosão dentária entre os sexos na infância. Outra explicação plausível é que os meninos têm maior hábito de consumo de bebidas ácidas em comparação às meninas (VARGAS FF, et al., 2011).

Além da dieta ácida, problemas gástricos podem resultar em erosão dentária devido ao contato da acidez do suco gástrico com a cavidade oral. O número de pacientes que possuem esse distúrbio tem aumentado de acordo com o estresse da vida moderna e, assim, tendem a ter redução na capacidade do tampão salivar, o que pode culminar com o aumento do potencial de erosão dos ácidos gástricos. O diagnóstico precoce é 
de suma importância para que os danos erosivos sejam minimizados. Raramente os pacientes e/ou responsáveis conseguem reconhecer os sinais iniciais da erosão dentária, por isso, o profissional deve ficar atento aos sinais clínicos e conscientizar os pacientes de que o consumo de bebidas ácidas tem sido apontado como significante fator de risco à erosão dental (SALAS MM, et al. 2015).

Existem fatores sociodemográficos, comportamentais e biológicos que podem estar associados à erosão dentária, mas seu diagnóstico clínico pode ser confundido com diversos tipos de desgastes. É imprescindível o conhecimento abundante acerca das características e manifestações clínicas para que a lesão seja identificada precocemente e o diagnóstico seja correto, possibilitando um tratamento ideal para o paciente, que vai envolver avaliação da higiene oral e dieta, identificação da etiologia e monitoramento do caso (LINNETT V e SEOW WK, 2001).

No caso relatado, concluiu-se que é fundamental esclarecer aos pacientes sobre as causas e consequências do desgaste erosivo, visando a preservação da estrutura dentária ainda em estágio precoce. Espera-se que, por meio do tratamento aplicado junto com a conscientização e compreensão sobre a necessidade de mudanças no estilo de vida, se possa impedir a progressão das lesões erosivas encontradas, evitando-se o comprometimento da dentição permanente, e mantendo-se a saúde e integridade da estrutura dentária ao longo da vida.

\section{REFERÊNCIAS}

1. AL-DLAIGAN YH, et al. The influence of frequently consumed beverages and snacks on dental erosion among preschool children in Saudi Arabia. Al- Nutrition Journal ,2017;16(80):1-6.

2. ALVES MSC, et al. Diagnóstico clínico e protocolo de tratamento do desgaste dental não fisiológico na sociedade contemporânea. Odontol. Clín. Cient., 2012; 11(3)247-251.

3. BARDOLIA P, et al. Prevalence and risk indicators of erosion in thirteen- to fourteen-year-olds on the Isle of Man. Caries Res 2010; 44(2):165-8.

4. BARTLETT D, et al. Basic Erosive Wear Examination (BEWE): a new scoring system for scientific and clinical needs. Clin Oral Invest, 2008;12(1):65-8.

5. BRANCO CA, et al. Dental erosion: diagnosis and treatment options. Rev Odontol UNESP. 2008;37(3):235-42.

6. BRUSIUS CD. Erosão dentária em adolescentes de Porto Alegre, RS [Dissertação]: Universidade Federal do Rio Grande do Sul. Faculdade de Odontologia. Programa de Pós-Graduação em Odontologia, 2013.

7. CARVALHO TS, et al. Consensus report of the European Federation of Conservative Dentistry: erosive tooth wear-diagnosis and management.Clin Oral Investig. 2015;19(7):1557-61.

8. COMAR LP, et al. Dental erosion: an overview on definition, prevalence, diagnosis and therapy. Brazilian Dental Science. 2013;16(1):6-17.

9. CORRÊA FNP, et al. Diagnóstico, prevenção e tratamento clínico da erosão dentária. Rev Assoc Paul Cir Dent. 2011;65(1):12-9.

10. CRISCI PCO, et al. Erosão dentária em crianças ansiosas: relato de caso clínico. J Health Sci Inst. 2014;32(1):86-9.

11. DUGMORE CR, ROCK WP. The prevalence of tooth erosion in 12-year-old children. Br Dent J 2004; 196(5):279-82.

12. EL AIDI H, et al. Dynamics of tooth erosion in adolescents: a 3-year longitudinal study. J Dent 2010; 38(2):131-7.

13. FONTES CL, et al. Abordagem da erosão dentária na clínica odontopediátrica: relato de casos. Rev. Odontol. 2016; 28(3): $262-9$.

14. KREULEN CM, et al. Systematic review of the prevalence of tooth wear in children and adolescents. Caries research. 2010;44(2):151-9.

15. LINNETT V, SEOW WK. Dental erosion in children: a literature review. Pediatric dentistry. 2001;23(1):37-43.

16. LUSSI A, JAEGGI T. Erosion. diagnosis and risk factors. Clinical oral investigations. 2008;12(1):5-13.

17. MAGALHÃES AC, et al. The influence of residual salivary fluoride Brazilian oral research, 2008;22(1):67-71.

18. MESSIAS DCF, et al. Estratégias para prevenção e controle da erosão dental. RGO Revista Gaúcha de Odontologia, 2011;59:07- 13.

19. RANDAZZO AR, et al. Erosão dentária por influência da dieta. Revisão da literatura e relato de caso clínico. Arquivo brasileiro de odontologia, 2006.

20. SALAS MM et al. Diet influenced tooth erosion prevalence in children and adolescents: Results of a meta-analysis and meta-regression. J Dent. 2015;43(8):865-75.

21. SANTANA MNS, et al. Prevalência de erosão dentária e fatores associados em uma população de escolares. Rev. odontol. UNESP, 2018;47(3):155-60.

22. USHIDA CE, CURY JA. Estudo in situ do efeito da frequência de ingestão de Coca-Cola na erosão do esmalte-dentina e reversão pela saliva. Revista de Odontologia da Universidade de São Paulo. 1999;13(2):127-34.

23. VARGAS FF, et al. Prevalence of tooth erosion and associated factors in 11-14-year-old Brazilian schoolchildren. J Public Health Dent 2011; 71(1):6-12. 